The result cues that the improvement of autism-like after with the treatment of activating brain and opening orifices scalp acupuncture therapy, no matter age.3. Compared before and after treatment in therapy group, there have significant difference in Language score. The result cues that activating brain and opening orifices scalp acupuncture therapy can significantly improve language function in autism children.

Conclusions Activating brain and opening orifices scalp acupuncture therapy can significantly improve the therapeutic effect, be better to improve the autism-like of children and raise the IO level and language ability.

\section{THE STUDY ON QUALITY OF LIFE OF CHILDREN WITH CEREBRAL PALSY}

doi:10.1136/archdischild-2012-302724.1524

Z Liu. Cerebral Palsy Rehabilitation Centre of Nanhai Hospital for Women and Children, Affiliated to Guangzhou University of Chinese Medicine Guangdong China, Foshan, China

Objective To study the quality of life of children with cerebral palsy.

Methods With the PedsOL4.0, 113 children with cerebral palsy were studied, and 52 children with common illness and 314 normal children were also studied and compared. With the PedsOL of school functioning, the children of these three groups who had been to school or kindergarten were also studied and compared.

Result The score of physiology functioning, communication functioning and total score of PedsOL in children with cerebral palsy were lower than those in the children with common illness and normal children. The difference has statistic significance. The score of emotional functioning in children with cerebral palsy was only lower than that in the normal children, the difference has statistic significance. The score of school functioning in children with cerebral palsy was significant lower than that in children with common illness and normal children $(\mathrm{P}<0.01)$.

Conclusion The quality of life of children with cerebral palsy is much lower than children with common illness and normal children. The illness has sever effect on the school functioning of children with cerebral palsy. Therefore, the whole improve of quality of life is the goal for the rehabilitation of children with cerebral palsy.

\section{MEASUREMENTS OF QUALITY OF LIFE FOR CHILDREN WITH CEREBRAL PALSY}

doi:10.1136/archdischild-2012-302724.1525

Z Liu. Cerebral Palsy Rehabilitation Centre of Nanhai Hospital for Women and Children, Affiliated to Guangzhou University of Chinese Medicine Guangdong China, Foshan, China

Aim The purpose of this article is to detect common measurements of quality of life (QOL) for children with cerebral palsy (CP). Method To review literatures which attempted to assess QOL in individuals with CP in MEDLINE.

Result Five measurements met the inclusion criteria, including PedsOL Generic Core Scales and Cerebral Palsy Module, CHO, KIDSCEEN, PODCI and CP-QOL.

Conclusion Five instruments consist of generic and conditionspecific version. Considering development of children both self and proxy report are available. All have acceptable clinical utility. However, further study should be given to comfirm their reliability and validity in assessing QOL of children with CP. There are some drawbacks using these instruments, including confusion of QOL, function and participation, failure to reflex real QOL for children, weak agreement between self and proxy report on social function and psychosocial domains.

\section{TREATING TYROSINEMIA TYPE 1: EXPERIENCE FROM KUWAIT}

doi:10.1136/archdischild-2012-302724.1526

SA Sadeq, H Bin Nakhi, N Al Naqeeb. Pediatrics, Adan Hospital, Kuwait, Kuwait

Hereditary tyrosinemia type1 (HT1) is an autosomal recessive disorder caused by deficiency of fumarylacetoacetase. Untreated tyrosinemia type I usually presents either with severe liver involvement or later with liver dysfunction and renal tubular dysfunction associated with growth failure and rickets. Early diagnosis and prompt treatment is essential for an improved prognosis. Combined treatment with nitisinone (NBTC) and a low-tyrosine diet has resulted in a greater than $90 \%$ survival rate.

We are describing the presentation, management and follow up of 6 kuwaiti patients having (HT1). The index case was the product of consanguineous marriage male who presented at five months of age with bleeding due to hepatic failure was found to have rickets, was given a trial of rectal NBTC but died at age of 7 months. All following siblings were screened for (HT1). Three children (two males and one female) out of four were affected. The other index case was the product of consanguineous marriage, a female died at age of 6 months with hepatic failure. Her father was the youngest brother of the first index case. Latter on, these parents had another affected female who was diagnosed on birth based on family history. All the patients were kept on analogue X phen tyr meth free milk beside breast feeding. Once diagnosis was confirmed they were started on NTBC $1 \mathrm{mg} / \mathrm{kg} /$ day twice daily. They were monitored regularly by complete blood count, liver and renal functions, blood amino acids, urine succinylacetone, ultrasound, CT abdomen, echocardiography and slit-lamp examination of the eyes.

\section{SEIZURE WORSENING CAUSED BY LOW SERUM VALPROATE LEVELS FROM AN INTERACTION BETWEEN VALPROATE AND MEROPENEM}

doi:10.1136/archdischild-2012-302724.1527

'S Biçer, 'GC Erdağ, ${ }^{2} \mathrm{C}$ Kocaman, ${ }^{1} \mathrm{~T}$ Giray, ${ }^{1} \mathrm{D}$ Çöl, ' $\mathrm{A}$ Vitrinel, 'Z Yalvaç. ${ }^{1} \mathrm{Child}$ Health and Diseases, Yeditepe Univ Medical Faculty; ' $C$ Child Neurology, Erdem Hospital, Istanbul, Turkey

Background Meropenem is a carbapenem antibiotic, has a broad spectrum of antimicrobial activity. Valproate is widely used in the treatment of epileptic seizures in children. In last years, low serum concentrations of valproate were reported in children and adults receiving concomitant administration with meropenem. We present an interaction of meropenem with valproate in an epileptic child, leading to seizure exacerbations owing to the rapidly-lowering of serum valproate concentration.

Case Report This report describes a 14-year-old epileptic girl inpatient who received concurrent treatment with meropenem and valproate for the treatment of lobar pneumonia, pleuresia and epilepsy, respectively. In this patient, an increase of seizure frequency and somnolence were observed after addition of meropenem on the treatment, and a rapid decline of valproate serum concentrations was observed after two dose meropenem. This event was the most likely cause of increased seizure frequency. The dosage of valproate was increased and meropenem was stopped. Two days later, the seizures stopped. Five days later, the serum valproate concentrations increased to three fold and four week later, they increase to therapeutic levels. The seizures were considered to be probably related to the concurrent administration of meropenem and valproate.

Conclusion To avoid drug interaction that reduces the serum concentration of valproate, meropenem should not be used in epileptic patients using valproate for the treatment of epilepsy. If concominant administration is essential, serum valproate levels should be monitorized frequently. 


\section{UTILITY OF RESEARCH OF CARDIAC BIOMARKERS FOR EARLY DIAGNOSIS OF ANTHRACYCLINE INDUCED CARDIOTOXICITY IN CHILDREN}

doi:10.1136/archdischild-2012-302724.1528

L Dimitiru. Medex Medical Center, lasi, Romania

Background Anthracycline-induced cardiotoxicity in children with malignant diseases often may be associated with significant changes in values of some cardiac biomarkers.

Objective To establish the value of research of cardiac biomarkers for early diagnosis of anthracycline induced cardiotoxicity.

Methods Patients, 46 children (aged 2 months - 18 years), treated with anthracyclines for malignant hemopathies. Control group: 20 healthy children without history of cardiac diseases. Patients and controls were investgated by: clinical exam, Doppler echocardiography (Echo), determination of plasma values of of cardiac biomarkers $\mathrm{BNP}$ (B natriuretic peptide) and cTnI (troponin).

Results Determination of cardiac biomarkers: *Increased plasma levels of BNP in $45.7 \%$ of patients, from a mean baseline of $89 \mathrm{ng} / \mathrm{ml}$ $(0-117 \mathrm{ng} / \mathrm{ml})$ to alue $240 \mathrm{ng} / \mathrm{ml}(0-810 \mathrm{ng} / \mathrm{ml}),{ }^{*}$ increasing cTnI values. plasma at $4.34 \%$ of cases, the initial values $<0.04 \mathrm{pg} / \mathrm{ml}$ to values $>0.04 \mathrm{pg} / \mathrm{ml}$ in 2 cases. Echo modifications: anthracycline induced cardiomyopathy or just only diastolic dysfunction of LV in majority of cases, often correlated with cumulative dose of anthracyclines. Biomarkers changes were correlated in most cases with the presence of clinical manifestations and echo modifications induced by anthracycline cardiotoxicity.

Conclusions Clinical or infraclinical manifestations of cardiotoxicity in children treated with anthracyclines is associated with increased levels of cardiac biomarkers: BNP and cTnI which is an useful marker for the.cardiotoxicity. Changes in this parameters appeared early than echo modifications in anthracicline induced cardiotoxicity and is necessary to systematic monitoring these parameters during and after cytostatic therapy.

\section{THE STUDY OF THE EFFECT OF CUMIN SEEDS EXTRACTS ON SOME PATHOGENIC BACTERIAL AGENTS}

doi:10.1136/archdischild-2012-302724.1529

${ }^{1} \mathrm{M}$ Motamedifar, ${ }^{2} \mathrm{G}$ Haghighati, ${ }^{2} \mathrm{~A}$ Yousefi Arvand. ${ }^{1}$ Department of Bacteriology and Virology, Shiraz AIDS Research Center (SHARC), Shiraz University of Medical Sciences; ${ }^{2}$ Department of Bacteriology and Virology, Medical School, Shiraz University of Medical Sciences, Shiraz, Iran

Background and Aims The antimicrobial effects of cumin (Cuminum cyminum L.), as an agent, have been mentioned previously. In this study the inhibitory effect of alcoholic and aqueous extracts on some Gram positive and Gram negative pathogenic bacteria was examined.

Methods Susceptibility of different standard bacterial strains to the prepared methanolic and aqueous extracts were studied by using disk diffusion method in comparison with penicillin and amikacin antibiotics. The bacteria studied were Escherichia coli (ATCC 25922), Pseudomonas aeruginosa (ATCC 27853), Kelebsiella pneumoniae (ATCC 10031), Bacillus cereus (ATCC 11778), meticillin susceptible Staphylococcus aureus (MSSA) ATCC 25923 and meticillin resistant Staphylococcus aureus (MRSA) ATCC 700698. The minimum inhibitory concentration (MIC) of extracts was also determined when necessary.

Results The Methanolic and aqueous extracts of Cuminum cyminum $(25-100 \mathrm{mg} / \mathrm{mL})$ caused growth inhibition zones of $S$. aureus with the diameter ranged ranging from 9.6-22.4 mm and 14-23.2 $\mathrm{mm}$ respectively. For MRSA growth inhibition zones ranged 13-23.4 $\mathrm{mm}$ for alcoholic extracts and 11-13.2 $\mathrm{mm}$ for the aqueous extract. For MSSA Staphylococci the methanolic extract caused inhibition diameter of $13 \mathrm{~mm}$ and aqueous extract with the diameter of 10-13 $\mathrm{mm}$. These extracts were ineffective on other investigated bacteria. MIC of alcoholic extracts for MRSA (700698) and MSSA (25923) was determined as $75 \pm 35.36 \mathrm{mg} / \mathrm{mL}$.

Conclusions Considering the antibacterial effects of alcoholic and aqueous extracts of cumin seeds on $S$. aureus observed in this investigation, continuing the research for studying the in-vivo effect of ant- microbial effects of Cunminum cyminum extracts on Staphylococci seems valuable.

\section{THE COMPARISON OF SEVERAL ANESTHETIC PREMEDICATION IN MAGNETIC RESONANCE IMAGINING (MRI) UNIT IN PEDIATRIC PATIENTS}

doi:10.1136/archdischild-2012-302724.1530

'V Tuna, ${ }^{2} \mathrm{MS}$ Çolak, ${ }^{2} \mathrm{H}$ Ulusoy, ${ }^{2} \mathrm{M}$ Kosucu. ${ }^{1}$ Trabzon Kanuni Eğitim ve Araştırma Hastanemize; ${ }^{2}$ Karadeniz Technical University, Faculty of Medicine, Trabzon, Turkey

We were aimed to compare the efficacy and safety of midazolam, tramadol and midazolam plus tramadol in magnetic resonance imaging for pediatric patients.

Undergoing MRI, for premedication, doses of midazolam 0.75 $\mathrm{mg} / \mathrm{kg}$, tramadol $3 \mathrm{mg} / \mathrm{kg}$ and midazolam $0,375 \mathrm{mg} / \mathrm{kg}+$ tramadol $1.5 \mathrm{mg} / \mathrm{kg}$ were administered. Undergoing MRI, these drugs were determined sedation, anxiety and separation scores, and compared the effects of exposure time, shots of the family and technician satisfaction, reliability and the side effects. Sixty pediatric patients undergoing MRI were selected prospective and randomly

From patients with ASA I-II risk groups, patients in 1-15 years olds were administered orally premedication before 30 minutes from sedation. After MRI, sedation, anxiety and separation scores in anesthesia recovery unit, the time of undergoing MRI and staying in recovery unit, the frequency of additional sedative drugs administration and the family and technician satisfaction were noted. In addition, follow up was vital parameters and side effects.

The efficiency of sedation in midazolam group was determined better than other groups. In tramadol group, there was not efficiently sedation. For this reason, the need of additional sedation was occurred and prolonged undergoing MRI time. In midazolam+tramadol group, anxiety was lesser than other groups. Comparing the other groups there was maximum nausea side effect in tramadol group and minimum in midazolam+tramadol group.

In conclusion, midazolam group, the best sedation was detected. The use of low dose combination of midazolam+tramadol was not effective for sedation but was decrease anxiety scores and adaptation of separation time in children undergoing MRI.

\section{DRUGS ADMINISTERED IN SICKLE CELL DISEASE VASO-OCLUSIVE CRISIS}

doi:10.1136/archdischild-2012-302724.1531

'MZ Delago, 'P Aliaga, ${ }^{2} \mathrm{M}$ Bandeira, ${ }^{3} \mathrm{CA}$ Teixeira, ${ }^{4} \mathrm{FJ}$ Ropero, ${ }^{5} \mathrm{~S}$ Taniguchi. ${ }^{1}$ Albert

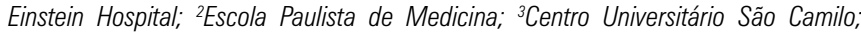
${ }^{4}$ UFABC; ${ }^{5}$ Basic Sciences, Albert Einstein Hospital, São Paulo, Brazil

Background and Aims The vascular occlusion of small vessels with blood cells in sickle cell disease leads to clinical manifestation such as pain crisis and organs' chronic damage.

Objective To study the drugs administered for pain treatment in patients with sickle cell disease vaso-oclusive episodes.

Methods Our study included 40 patients with sickle cell disease with mean age $8.95 \pm 0.6$ years old under pain treatment.

Results Clinical manifestations observed were vaso-oclusive crisis $(100 \%)$, splenomegaly $(17.5 \%)$, stroke $(5 \%)$,icteric syndrome $(5 \%)$, acute chest syndrome (2.5\%), dyspnea (32.5\%).

Patients with mild pain $(10 \%)$ received dipyrone $22 \mathrm{mg} / \mathrm{kg}$ every 6 hours (100\%) and tramadol $1.1 \mathrm{mg} / \mathrm{kg} \mathrm{q} 4-8 \mathrm{~h}(75 \%)$. 